\title{
Study of Compounds Suppressing Free Radical Generation from UV-Exposed Ketoprofen
}

\author{
Ayako Nakajima, ${ }^{*, a}$ Maiko TAhara, ${ }^{a}$ Yoshihiro Yoshimura, ${ }^{b}$ and Hiroyuki NaKaZawa ${ }^{a}$ \\ ${ }^{a}$ Department of Analytical Chemistry, Hoshi University; 2-4-41 Ebara, Shinagawa-ku, Tokyo 142-8501, Japan: and \\ ${ }^{b}$ Nihon Pharmaceutical University; 10281 Komuro, Ina-cho, Adachi-gun, Saitama 362-0806, Japan. \\ Received March 14, 2007; accepted July 17, 2007; published online July 20, 2007
}

Ketoprofen [(RS)-2-(3-benzoylphenyl)propanoic acid] is widely used for the treatment of inflammatory diseases and musculoskeletal injury. However, there is concern regarding its potential for photosensitization as a side effect. Free radicals and active oxygen species generated from ketoprofen on exposure to ultraviolet (UV) light have been implicated in phototoxicity and photosensitization. In this study, we examined the suppressing ability of some compounds for the free radicals and active oxygen species generated by the photodynamic reaction of ketoprofen, to determine a new resist of photosensitization by ketoprofen. Eight compounds, including six known free radical scavengers were individually mixed with ketoprofen, and the mixtures were exposed to UV. Then, the free radicals and the active oxygen species were determined by the electron spin resonance spectrometry to estimate suppressing and scavenging ability of compounds. The compounds that show promise in suppressing superoxide anion generation from UV-exposed ketoprofen were further evaluated using the on-line photo-irradiated superoxide anion detection system. It was confirmed that quercetin, a flavonoid, strongly suppresses the generation of free radicals and active oxygen species from UV-exposed ketoprofen. The experiments using the experimental formulation of an adhesive skin patch of ketoprofen containing quercetine and the Chemiluminescence analyzer (CLA) indicated that quercetin has high potential for use as an excipient in ketoprofen ointments to suppress phototoxicity and photosensitization by ketoprofen.

Key words ketoprofen; photosensitization; suppressor; active oxygen species; quercetin

Photosensitization, phototoxicity or photoallergy is induced on exposure to sunlight after internal or external administration of ketoprofen. Ketoprofen is absorbed via the gastrointestinal tract and is carried to the near surface of skin by the circulation where it is directly exposed to sunlight. Free radicals were reported to damage DNA ${ }^{1-4)}$ and to induce hemolysis, ${ }^{1,5-7)}$ and active oxygen species, superoxide anion and singlet oxygen generated from ultraviolet (UV)exposed ketoprofen were found to contribute to dermatitis. ${ }^{8)}$ Although the free radical species were speculated to be benzophenone, ${ }^{9-12)}$ benzoyl radical, ${ }^{13)}$ carbonyl radical ${ }^{14)}$ and superoxide anion, ${ }^{15}$ their true identities remain unknown to this day. We elucidated the pathways for the photo-degradation of ketoprofen and the generation of free radicals in our previous study. ${ }^{16)}$ It was recently reported that some compounds scavenge free radicals that are thought to contribute to illness, carcinogenicity and senescence. ${ }^{17)}$ In this study, we examined the suppressing and scavenging effects of some compounds on the free radicals generated by the photodynamic reaction of ketoprofen, to determine a new resist of photosensitization by ketoprofen. Eight compounds, including allopurinol, $l$-histidine, oxybenzone, chloropromazine, $\alpha$ tocopherol and quercetine, were used in the experiments. $l$ Histidine is known to be a scavenger of hydroxyl radical ${ }^{18)}$ and singlet oxygen. ${ }^{19)}$ Oxybenzone is widely used as an additive in cosmetics and foods to absorb UV. Chloropromazine is used to treat anxiety, stress, nausea, vomiting and other symptoms caused by integration disorder syndrome, manic psychosis or nervous disease. $\alpha$-Tocopherol and quercetin are known as a free radical scavenger.

\section{Experimental}

Reagents and Materials Ketoprofen was obtained from Sigma-Aldrich Japan (Tokyo, Japan). ( \pm )-Epinephrine was purchased from Sigma-Aldrich (St. Louis MO, U.S.A.). 5,5-Dimethyl-1-pyroline- $N$-oxide (DMPO) was ob- tained from Dojindo Laboratories (Kumamoto, Japan). Quercetin was purchased from Tokyo Kasei Co., Ltd. (Tokyo, Japan), and $\alpha$-tocopherol, chloropromazine, $l$-histidine, allopurinol and flavone were obtained from Wako Pure Chemical Industries (Osaka, Japan). Phenothiadine was purchased from Kanto Chemical Co., Ltd. (Tokyo, Japan) and oxybenzone was obtained from Sigma-Aldrich Japan (Tokyo, Japan). Acetonitrile and methanol of HPLC grade, ammonium acetate, and dimethyl sulfoxide (DMSO) were purchased from Wako Pure Chemical Industries (Osaka, Japan). Laboratory water was purified with a Milli-Q gradient A10 Elix with an EDS polisher system (Millipore, Bedford, MA, U.S.A.).

Equipment The OPSD system consisted of intelligent pumps, LC10AD (Shimadzu, Kyoto, Japan) and L-6300 (Hitachi, Tokyo, Japan); a column oven, CTO-6A (Shimadzu, Kyoto, Japan); and a UV-VIS detector, SPD-6AV (Shimadzu, Kyoto, Japan). The light source was a chemical lamp, FL6BL (6W, Toshiba, Tokyo), and the Teflon polytetrafluoroethylene (PTFE) tube was $2.5 \mathrm{~m} \times 0.5 \mathrm{~mm}$ I.D. (Nishio, Tokyo, Japan). The ESR system used was JES-RE1X (JEOL, Tokyo, Japan) and the light source was a UV lamp, GL-6 (6W, maximum wavelength $254 \mathrm{~nm}$ ) (Panasonic, Tokyo, Japan). The UV-VIS spectrophotometer used was UV-1600PC (Shimadzu, Kyoto, Japan). The Chemiluminescence analyzer (CLA) used was CLA-FS1 (Tohoku Electron, Miyagi, Japan). An ultraviolet lamp of CLA was UV GL-25, MINERALLIGHT LAMP, MULTIBAND UV-254/366nm (115Volts, $60 \mathrm{~Hz}$, 0.16 Amps) (UVP. INC, CA 91778, U.S.A.).

The UV-VIS Spectrophotometry Ketoprofen $(10 \mu \mathrm{g} / \mathrm{ml})$ was dissolved in methanol/water $(1: 1)$ solution for UV/VIS measurement. Quercetin was dissolved in methanol, oxybenzone was dissolved in methanol/water $(1: 1)$, flavone and phenothiazine were each dissolved in acetonitrile, $\alpha$-tocopherol was dissolved in ethanol, and chloropromazine, allopurinol and $l$-histidine were each dissolved in water to make $100 \mu \mathrm{m}$ solutions. Each solution and the corresponding solvent were transferred into a $1 \mathrm{~cm} \times 1 \mathrm{~cm}$ quartz cell for UV-VIS measurement. An absorption spectrum of 190 to $900 \mathrm{~nm}$ was obtained for each solution.

Determination of Photodecomposition Curve Ketoprofen $(200 \mu \mathrm{M})$ was dissolved in methanol/water $(1: 1)$ solution (Solution A). Quercetin was dissolved in methanol, oxybenzone was dissolved in methanol/water $(1: 1)$, flavone and phenothiazine were each dissolved in acetonitrile, $\alpha$-tocopherol was dissolved in ethanol, and chloropromazine, allopurinol and $l$-histidine were each dissolved in water to make $100,200,400,1000$ and $2000 \mu \mathrm{M}$ solutions (Solution B). One milliliter of Solution A and $1 \mathrm{ml}$ of Solution B were mixed and exposed to UV for $0,5,10,20,30,45$ and $60 \mathrm{~min}$ respectively. Ten microliters of each exposed solution was injected into the HPLC 
system under the following conditions: Mobile phase: acetonitrile/methanol/ $20 \mathrm{~mm}$ ammonium acetate $(30: 50: 20)$; flow rate: $1.0 \mathrm{ml} / \mathrm{min}$; column: GLPACK Nucleosil $250 \mathrm{~mm} \times 4.6 \mathrm{~mm}$ I.D.; column temperature: $40^{\circ} \mathrm{C}$; and detection wavelength: $372 \mathrm{~nm}$ (quercetin), $290 \mathrm{~nm}$ (flavone), $254 \mathrm{~nm}$ (ketoprofen, chloropromazine, $l$-histidine and oxybenzone) or $252 \mathrm{~nm}$ (phenothiazine and allopurinol).

The ratio of peak area of ketoprofen and 2,3-bis-(3-benzoylphenyl)butane (photo-degradation product A) at each irradiation time to the peak area of ketoprofen at initial time was calculated. These ratios were plotted versus the irradiation times to make the photodecomposition curve of ketoprofen and the generation curve of the photo-degradation product $\mathrm{A} .{ }^{16,20,21)}$

Off-Line Photo-Irradiation/ESR Experiments. Sample Preparation Ketoprofen, oxybenzone, $\alpha$-tocopherol, flavone and phenothiazine were each dissolved in acetonitrile, quercetin and allopurinol were each dissolved in DMSO/water $(1: 1)$, and chloropromazine and $l$-histidine were each dissolved in water. Ketoprofen solution ( $2 \mathrm{~mm}, 0.15 \mathrm{ml}), 0.15 \mathrm{ml}$ of $20 \mathrm{~mm}$ compound solution and $5 \mu \mathrm{l}$ of DMPO were mixed, exposed to UV for $0,5,10$ 30 , or $60 \mathrm{~min}$, and used in ESR measurements.

ESR Measurement Conditions ESR spectra were obtained under the following conditions: center field, $336.5 \mathrm{mT}$; sweep time, $5 \mathrm{~s}$; sweep width, $\pm 5 \mathrm{mT}$; field modulation width, 0.063; receiver gain, 500; and time constant, $0.03 \mathrm{~s}$.

OPSD System. Sample Preparation Ketoprofen $(10 \mathrm{~mm})$ and each compound solution were respectively prepared. Ketoprofen was dissolved in acetonitrile/water $(1: 1)$, quercetin was dissolved in DMSO and $\alpha$-tocopherol was dissolved in acetonitrile. Epinephrine $(100 \mu \mathrm{M})$ in acetonitrile/ water $(1: 1)$ was prepared as radical detecting reagent solution. Ketoprofen solution $(10 \mu \mathrm{l})$, each compound solution $(10 \mu \mathrm{l})$, and epinephrine solution $(10 \mu \mathrm{l})$ were mixed (Solution $\mathrm{C})$. Solution $\mathrm{C}(7.5 \mu \mathrm{l})$ was injected into the on-line system. Ten microliters each of ketoprofen solution and epinephrine solution was mixed to make a blank solution and $5 \mu \mathrm{l}$ of this blank was injected.

Equipment The column-switching on-line HPLC system was equipped with a light exposing cell, a six-port switching valve, a make-up pump, an analytical HPLC and a UV/VIS detector. The light irradiation equipment consisted of a reaction coil and the chemical lamp, FL6BL: Teflon tube $(1.5 \mathrm{~m})$ was knitted and coiled around a tube of quartz $(15 \mathrm{~cm}$ and $2.5 \mathrm{~cm}$ I.D.), and the chemical lamp was inserted into this quartz tube to prevent deterioration of the Teflon tube by the heat of the lamp. This light exposing equipment was covered with an aluminum-coated box $(20 \times 27 \times 20 \mathrm{~cm})$ to protect from room light. ${ }^{16)}$

Peak Detection Solution $C$ was injected into the system and exposed by the chemical lamp. The product of the reaction of the superoxide anion with epinephrine and the photo-degradation products were separated and detected under the following HPLC conditions.

HPLC Conditions: Mobile phase: acetonitrile/methanol/20 mM ammonium acetate $(30: 50: 20)$; flow rate: $1.0 \mathrm{ml} / \mathrm{min}$; column: GL-PACK Nucleosil $250 \mathrm{~mm} \times 4.6 \mathrm{~mm}$ I.D.; column temperature: $40^{\circ} \mathrm{C}$; and detection wavelength: $480 \mathrm{~nm}$.

Determination of Superoxide Anion Suppressing Ability A mixture of epinephrine, the compound and ketoprofen was pumped into the photoreaction cell, where it was exposed to the chemical lamp. Epinephrine reacted with the superoxide anion to produce adrenochrome, a stable compound that exhibits maximum absorption at $480 \mathrm{~nm}$ (Chart 1). The derived compound and the photo-degradation products were pumped into the HPLC column by column switching and their peaks were separated and detected by the HPLC system. If the compound has superoxide anion suppressing ability, the HPLC peak of adrenochrome would be diminished. Therefore, the $\%$ decrease in peak area or height of adrenochrome is an indication of the ability of the compound to suppress the generation of superoxide anion.

Determination for Singlet Oxygen Suppressing Ability of Quercetin by CLA The experimental ketoprofen formulation of the adhesive skin

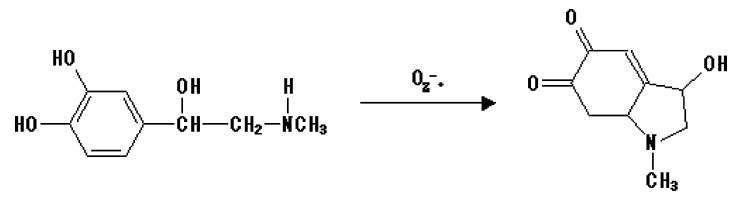

Epinephrine

adrenochrome

Chart 1. The Chemical Reaction of Epinephrine and Superoxide Anion to Produce Adrenochrome patch was prepared based on the recipe of the ethical drug, using quercetin instead of oxybenzone (the quercetin formulation). $0.30 \mathrm{~g}$ of ketoprofen and $0.36 \mathrm{~g}$ of quercetin were contained in $100 \mathrm{~g}$ of ointment's components. The other excipients were $N$-methyl-2-pyrolidone, $l$-menthol, partially neutralized polyacrylate, sodium polyacrylate, carmellose sodium, gelatin, hydroxyl aluminum, aluminum sulfate, polyoxyethylene sorbitan triolate, glycerin, edetate trisodium, thymol, silicic acid anhydride and $\mathrm{pH}$ adjuster. The control formulation was made by subtracting quercetin from the above quercetin formulation. These patches were cut and each of their pieces was placed on the stainless and flat-bottomed sample container $(10 \mathrm{~mm} \times 50 \mathrm{~mm}$ i.d.) of the CLA and irradiated by $366 \mathrm{~nm}$ of light beam for $5 \mathrm{~s}$ in air atmosphere, at room temperature. The chemiluminescence (CL) spectrum was obtained through a photo filter $330-700 \mathrm{~nm}$.

\section{Results}

Ability of Six Compounds to Suppress Photoreaction of Ketoprofen The ability of the six compounds to suppress the photoreaction of ketoprofen, namely, allopurinol (A) chloropromazine (B), l-histidine (C), oxybenzone (D), quercetin (E), and $\alpha$-tocopherol (F), was evaluated based on the following requirements: (1) suppress photodecomposition of ketoprofen (umbrella effect), (2) quench free radicals generated from UV-exposed ketoprofen, (3) does not generate free radicals due to UV exposure, (4) does not decompose due to UV exposure. (1) and (4) were elucidated from the photodecomposition curves of the mixtures of ketoprofen and each of the compounds. (2) and (3) were determined by ESR experiments using mixtures of ketoprofen and each of the compounds and the solutions of each of the compounds alone, exposed to UV. The results of evaluation of the suppressing ability of the six compounds is summarized in Table 1. The check mark " $\checkmark$ " indicates that the compound satisfied the requirement, and the cross mark " $X$ " indicates that the compound did not satisfy the requirement. The chemical structures and chemical names of the six compounds are shown in Chart 2.

Ability of Basic Skeleton of Quercetin and Chloropromazine to Suppress Photoreaction of Ketoprofen According to the above experimental results, chloropromazine and quercetin were expected to be strong suppressors of the photoreaction of ketoprofen. Although it can scavenge free radicals, $\alpha$-tocopherol could not suppress the photodecomposition of ketoprofen. The decomposition curves of ketoprofen and the generation curves of photo-degradation product $A$ when chloropromazine, $\alpha$-tocopherol and quercetin were used are shown in Figs. 1, 2 and 3, respectively. The ESR spectrum of UV-exposed ketoprofen is shown in Fig. 4, and

Table 1. The Results of Determination of Ability to Suppress Photoreaction of Ketoprofen for Six Compounds

\begin{tabular}{lcccc}
\hline \hline \multirow{2}{*}{ Compound } & \multicolumn{5}{c}{ Requirement } \\
\cline { 2 - 5 } & $(1)$ & $(2)$ & $(3)$ & $(4)$ \\
\hline Allopurinol (A) & $\checkmark$ & $\times$ & $\times$ & $\checkmark$ \\
Chloropromazine (B) & $\checkmark$ & $\checkmark$ & $\checkmark$ & $\times$ \\
l-Histidine (C) & $\times$ & $\times$ & $\times$ & $-^{a}$ \\
Oxybenzone (D) & $\checkmark$ & $\times$ & $\checkmark$ & $\checkmark$ \\
Quercetin (E) & $\checkmark$ & $\checkmark$ & $\checkmark$ & $\checkmark$ \\
$\alpha$-Tocopherol (F) & $\times$ & $\checkmark$ & $\checkmark$ & $-^{a}$
\end{tabular}

(1) Umbrella effect: suppress photodecomposition of ketoprofen, (2) quench free radicals generated from UV-exposed ketoprofen, (3) does not generate free radicals due to UV exposure, (4) does not decompose due to UV exposure. a) "--" means this compound was not detected under the HPLC condition due to weak UV absorption. 
<smiles>Oc1ncnc2[nH]ncc12</smiles><smiles>CCCN1c2ccccc2Sc2ccc(Cl)cc21</smiles><smiles>NC(N)(Cc1c[nH]cn1)C(=O)O</smiles><smiles>COc1ccc(C(=O)c2ccccc2)c(O)c1</smiles>

D<smiles>CC1=C2CCC(CCC(C)CCC(C)CCCC(C)C)CC(O2)C(C)C1C</smiles>

F<smiles>O=c1c(=O)c2c(Br)cc(Br)cc2oc2cc(Cl)ccc12</smiles>

$\mathrm{E}$

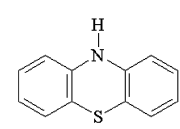

G

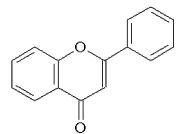

$\mathrm{H}$
A: 1H-Pyrazolo[3,4-d]pyrimidin-4-ol (allopurinol)
B: 2-Chloro-N,N-dimethyl-10H-phenothiazine-10-propanamine (chlorpromazine)
C: (S)- $\alpha$-amino-1H-imidazole-4-propanoic acid (L-histidine)
D: 2-Hydroxy-4-methoxybenzophenone (oxybenzone)
E: 2-(3,4-Dihydroxyphenyl)-3,5,7-trihydroxy-4H-1-benzopyran-4-one (quercetin)
F: 2,5,7,8-Tetramethyl-2-(4,8,12-trimethyltridecyl)chroman-6-ol ( $\alpha$-tocopherol)
G: 10H-Phenothiazine
H: 2-Phenyl-4H-1-benzopyran-4-one (flavone)

Chart 2. The Chemical Structures and Chemical Names of the Six Compounds Used to Confirm Their Ability to Suppress Photoreaction of Ketoprofen
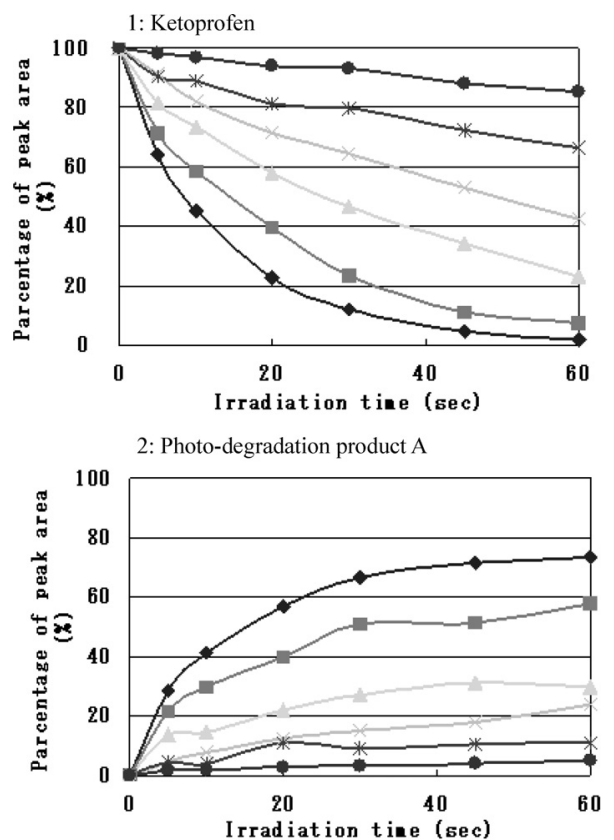

Ratio of mol conc. of chloropromazine (C) versus ketoprofen $(\mathrm{K})$

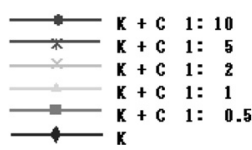

Fig. 1. Degradation Curve of Ketoprofen and Generation Curve of PhotoDegradation Product A, in the Presence of Chloropromazine
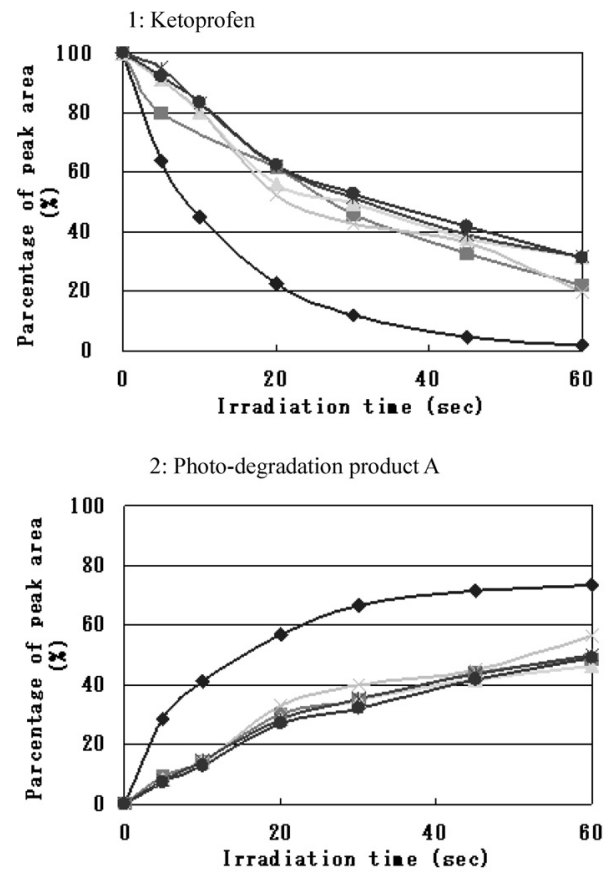

Ratio of mol conc. of $\alpha$-tocopherol (T) versus ketoprofen (K)

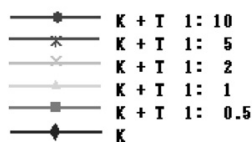

Fig. 2. Degradation Curve of Ketoprofen and Generation Curve of PhotoDegradation Product A, in the Presence of $\alpha$-Tocopherol
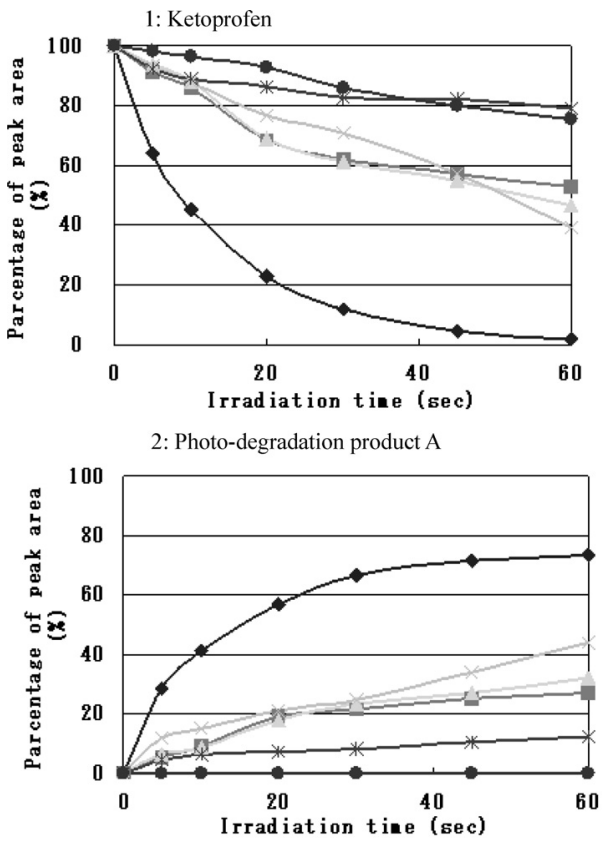

Ratio of mol conc. of quercetin $(\mathrm{Q})$ versus ketoprofen $(\mathrm{K})$

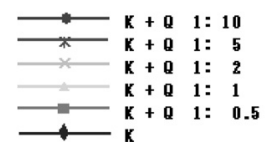

Fig. 3. Degradation Curve of Ketoprofen and Generation Curve of PhotoDegradation Product A, in the Presence of Quercetin 
the ESR spectra of the UV-exposed mixtures of ketoprofen and each of chloropromazine, $\alpha$-tocopherol and quercetin are provided in Figs. 5, 6 and 7, respectively. Further investigations of the chemical structures of chloropromazine and quercetin were carried out. The suppressing ability of their basic skeleton compounds, phenothiazine and flavone, whose chemical structures are presented in Chart 2, was evaluated as well, and the results are summarized in Table 2. The ESR spectra of phenothiazine and flavone are presented in Figs. 8 and 9 .

The OPSD System Determines Suppressing Ability of Quercetin and $\alpha$-Tocopherol The ability to suppress superoxide anion generation from UV-exposed ketoprofen was determined from the decrease in peak height of adrenochrome, which is a product of the reaction of superoxide anion with epinephrine. Fluctuations of the peak heights of adrenochrome, ketoprofen and photo-degradation product A were examined when ketoprofen was irradiated in the

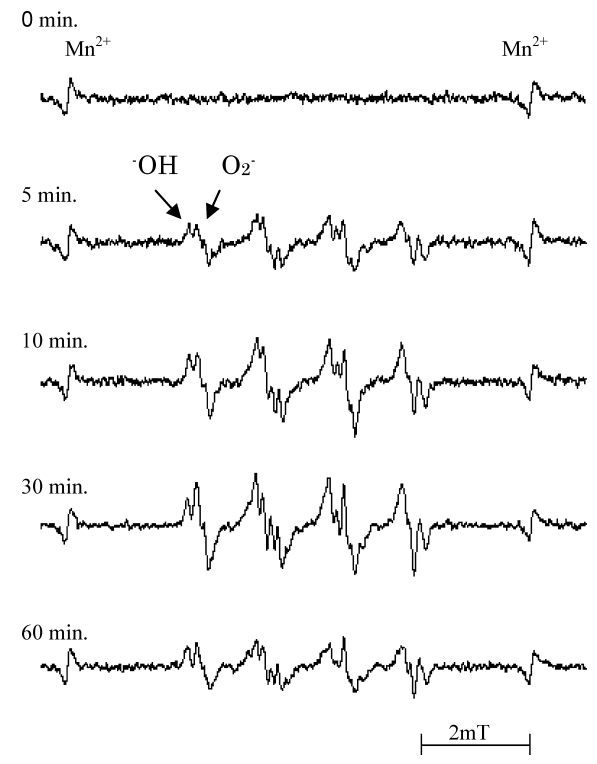

Fig. 4. The ESR Spectra of UV-Irradiated Ketoprofen

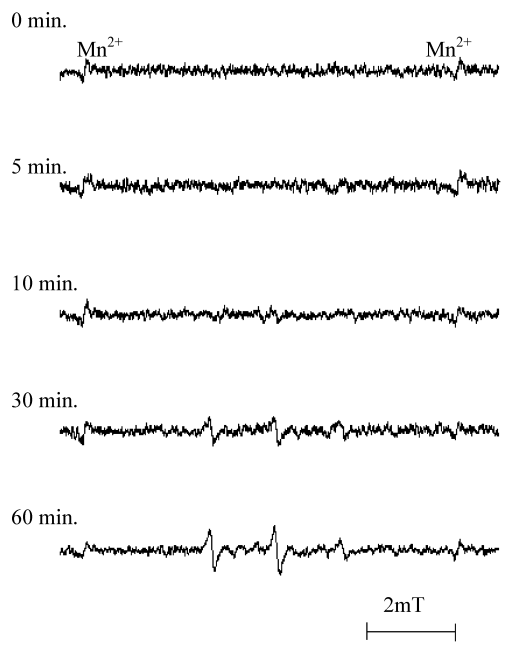

(1) presence of quercetin or $\alpha$-tocopherol. The peak height when ketoprofen alone was irradiated was considered to be $100 \%$. The results are summarized in Table 3 and the spectra are presented in Fig. 10.

The CLA Determines Suppressing Ability of Quercetin in the Formulation The CL spectra for the quercetin formulation and the control formulation are shown in Fig. 11. In the CL spectrum for the control formulation, a strong CL peak was detected at around $35 \mathrm{~s}$, and the maximum intensity of CL was 70000 counts/s. On the other hand, the maximum intensity of CL peak was diminished to 2800 counts/s in the CL spectrum for the quercetin formulation.

\section{Discussion}

Although the six compounds investigated in this study are acknowledged as free radical scavengers, three of them do not have the ability to quench free radicals and active oxygen species generated from UV-exposed ketoprofen. The ESR results indicated that allopurinol itself generates free radicals when exposed to UV. Therefore, allopurinol could not be used to prevent photosensitization by ketoprofen although it is a strong suppressor of the photodecomposition of ketoprofen. $l$-Histidine hardly absorbs UV due to its chemical structure; thus, it cannot suppress the decomposition of ketoprofen. The ESR results indicated that $l$-histidine neither suppressed the generation of free radicals and active oxygen species from UV-exposed ketoprofen nor quenched them, although $l$-histidine is known to be a scavenger of hydroxyl radical and singlet oxygen. It was considered that allopurinol and $l$-histidine were affected by UV light energy and their scavenging activity was obstructed by unexpected photoreactions. In the fact, even ketoprofen was reported to be a scavenger of hydroxyl radicals. ${ }^{22)}$ It was confirmed that oxybenzone does not quench free radicals and active oxygen species generated from UV-exposed ketoprofen, although it suppresses the decomposition of ketoprofen. Nevertheless, oxybenzone does not induce photosensitivity when used as UV absorber in cosmetics. According to the results of screening (Table 1), three promising compounds, chloropromazine, quercetin and $\alpha$-tocopherol, were selected for further investi-
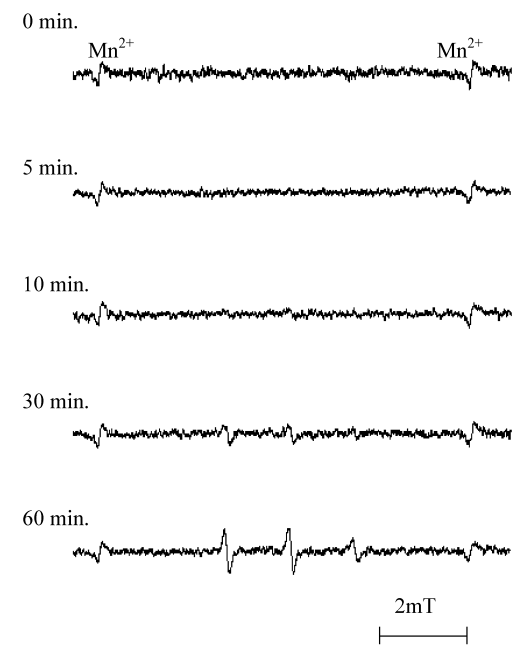

(2)

Fig. 5. ESR Spectra of UV Irradiated Sole Solution of Chloropromazine and UV Irradiated Mixture Solution of Ketoprofen and Chloropromazine (1) UV irradiated sole solution of chloropromazine, (2) UV irradiated mixture solution of ketoprofen and chloropromazine. 


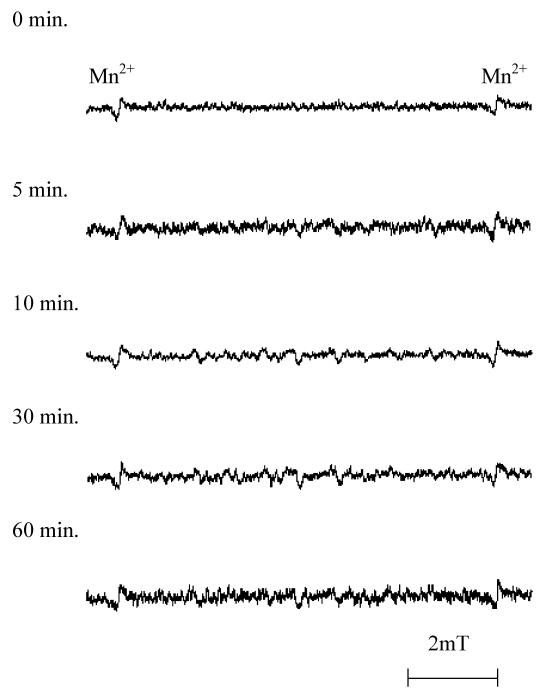

(1)

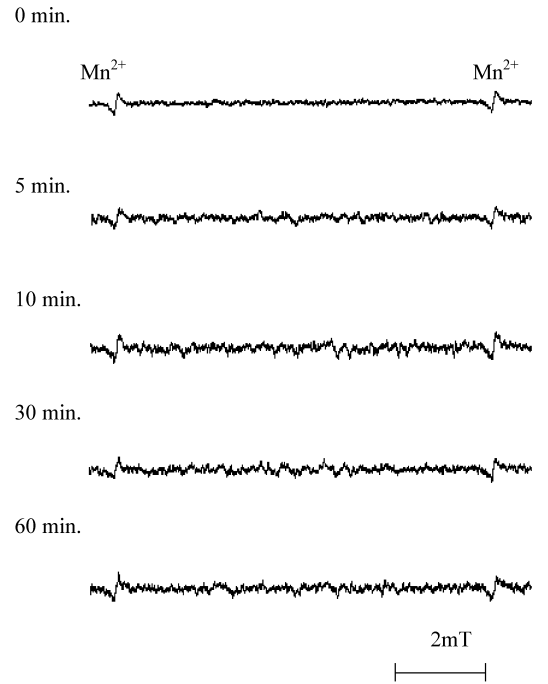

(2)

Fig. 6. ESR Spectra of UV Irradiated Sole Solution of $\alpha$-Tocopherol and UV Irradiated Mixture Solution of Ketoprofen and $\alpha$-Tocopherol (1) UV irradiated sole solution of $\alpha$-tocopherol, (2) UV irradiated mixture solution of ketoprofen and $\alpha$-tocopherol.

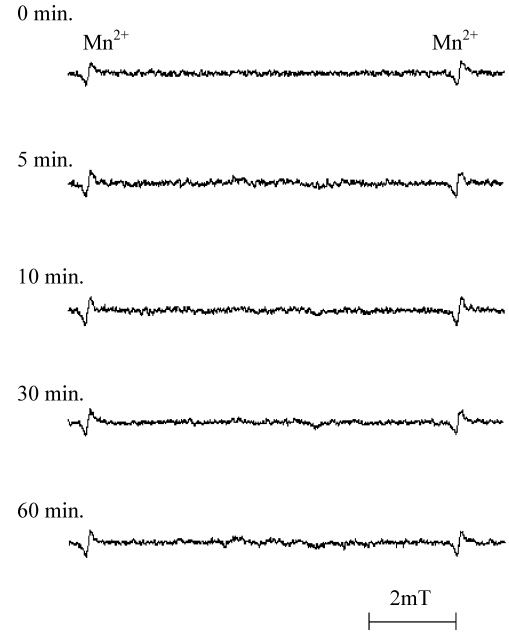

(1)

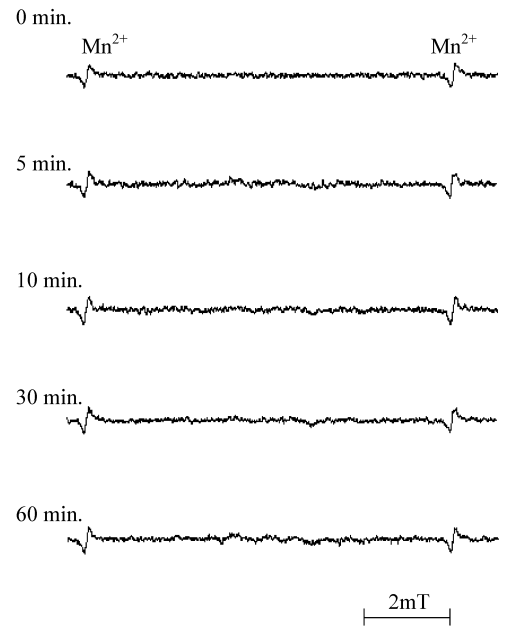

(2)

Fig. 7. ESR Spectra of UV Irradiated Sole Solution of Quercetin and UV Irradiated Mixture Solution of Ketoprofen and Quercetin (1) UV irradiated sole solution of quercetin, (2) UV irradiated mixture solution of ketoprofen and quercetin.

Table 2. The Results of Determination of Ability to Suppress Photoreaction of Ketoprofen for Phenothiazine and Flavone

\begin{tabular}{lcccc}
\hline \hline \multirow{2}{*}{ Compound } & \multicolumn{5}{c}{ Requirement } \\
\cline { 2 - 5 } & $(1)$ & $(2)$ & $(3)$ & (4) \\
\hline Phenothiazine & $\checkmark$ & $\times$ & $\times$ & $\checkmark$ \\
Flavone & $\checkmark$ & $\times$ & $\checkmark$ & $\checkmark$ \\
\hline
\end{tabular}

(1) Umbrella effect: suppress photodecomposition of ketoprofen, (2) quench free radicals generated from UV-exposed ketoprofen, (3) does not generate free radicals due to UV exposure, (4) does not decompose due to UV exposure.

gation.

Chloropromazine The results of HPLC analyses of UVexposed chloropromazine solution revealed that chloropromazine was decomposed in a manner similar to ketoprofen. Chloropromazine, however, suppressed the decomposition of ketoprofen and the generation of photo-degradation product
A (Fig. 1). This suppressing ability was increased with increasing concentration of chloropromazine relative to ketoprofen. The ESR spectrum of the mixture of chloropromazine and ketoprofen demonstrated that chloropromazine quenched free radicals and superoxide anions generated from ketoprofen. Although the ESR spectrum of UV-exposed chloropromazine solution indicated slight generation of free radicals at $60 \mathrm{~min}$, it is acceptable to satisfy the requirement No. 3 (Fig. 5). These results indicated that chloropromazine may be a superior suppressor of free radical generation by the photoreaction of ketoprofen. However, it was also confirmed that the basic skeleton of chloropromazine, phenothiazine, did not quench free radicals generated from UV-exposed ketoprofen, and that phenothiazine itself more likely generated free radicals and superoxide anions (Fig. 8). It was therefore considered that the free radical quenching ability of chloropromazine is not based on its basic skeleton and that chloropromazine poses the risk of photosensitivity due to the 


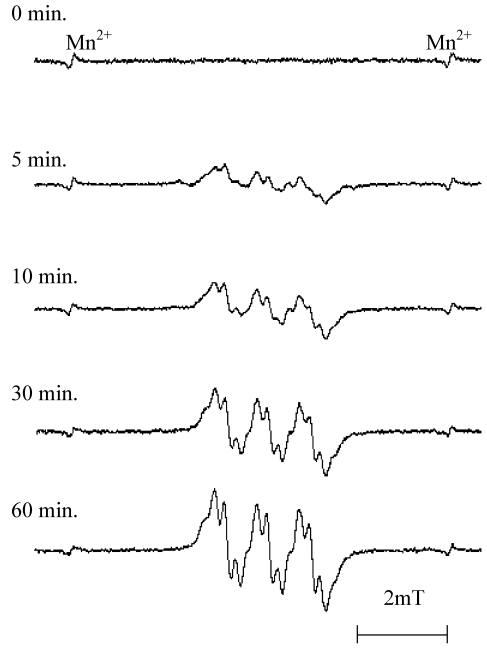

(1)
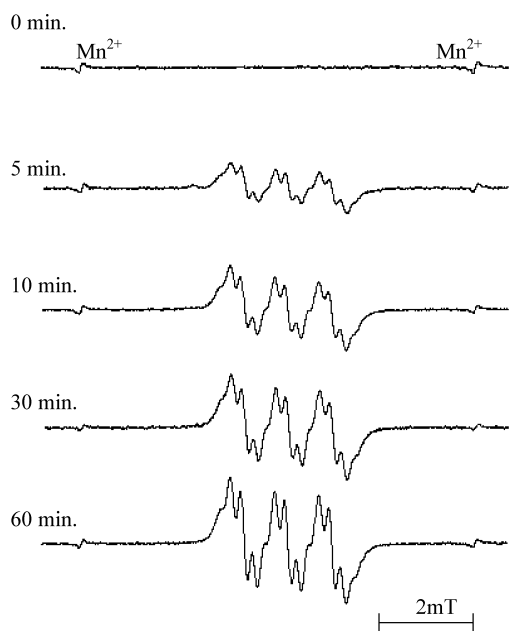

(2)

Fig. 8. ESR Spectra of UV Irradiated Sole Solution of Phenothiazine and UV Irradiated Mixture Solution of Ketoprofen and Phenothiazine

(1) UV irradiated sole solution of phenothiazine, (2) UV irradiated mixture solution of ketoprofen and phenothiazine.

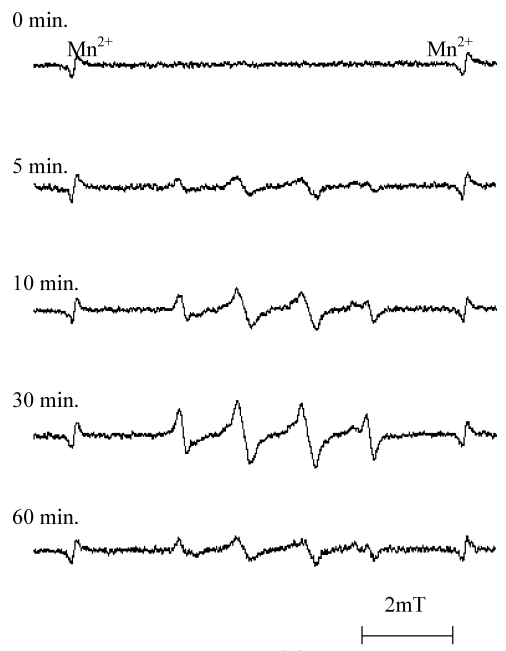

(1)

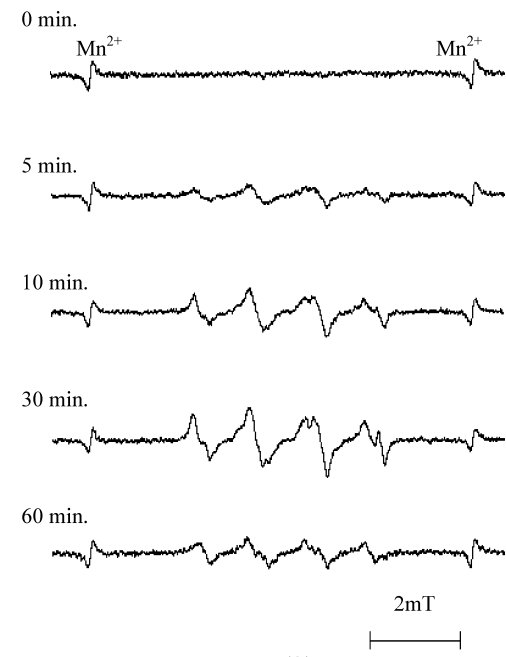

(2)

Fig. 9. ESR Spectra of UV Irradiated Sole Solution of Flavone and UV Irradiated Mixture Solution of Ketoprofen and Flavone

(1) UV irradiated sole solution of flavone, (2) UV irradiated mixture solution of ketoprofen and flavone.

Table 3. Changes in Peak Heights of Adrenochrome, Ketoprofen and Photo-Degradation Product A in OPSD Experiments

\begin{tabular}{lccc}
\hline \hline \multirow{2}{*}{ Injected compound } & \multicolumn{3}{c}{ Peak identification } \\
\cline { 2 - 4 } & Aadrenochrome & Ketoprofen & $\begin{array}{c}\text { Photo-degradation } \\
\text { product A }\end{array}$ \\
\hline Ketoprofen alone & $100 \%$ & $100 \%$ & $100 \%$ \\
Quercetin+ketoprofen & $63.3 \%$ & $142.0 \%$ & $69.4 \%$ \\
$\alpha$-Tocopherol+ketoprofen & $95.9 \%$ & $75.7 \%$ & $73.4 \%$ \\
\hline
\end{tabular}

phenothiazine structure.

$\alpha$-Tocopherol According to the results of decomposition analysis, only $30 \%$ of ketoprofen remained after UV exposure for $60 \mathrm{~s}$, when $\alpha$-tocopherol was added. Although $\alpha$-tocopherol suppresses the decomposition of ketoprofen slightly and the generation of photo-degradation product A, this suppressing ability was not enhanced even when its concentration was increased (Fig. 2). This means that this suppressing ability could not be attributed to the umbrella effect. ESR experiments demonstrated that $\alpha$-tocopherol scavenged free radicals generated from UV-exposed ketoprofen, and no free radicals were generated from $\alpha$-tocopherol even though it was exposed to UV (Fig. 6). In OPSD experiments, the peak height of adrenochrome was decreased slightly and the peak height of ketoprofen showed no increase. However, it was clear that $\alpha$-tocopherol suppressed the generation of photodegradation product $\mathrm{A}$, which means that $\alpha$-tocopherol weakly suppresses the generation of free radicals and the decomposition of ketoprofen, but strongly quenches free radicals generated from UV-exposed ketoprofen (Fig. 10, Table 3).

Quercetin Among the compounds studied, quercetin most effectively suppressed the decomposition of ketoprofen and the generation of photo-degradation product $\mathrm{A}$, because $80 \%$ of ketoprofen remained after UV exposure for $60 \mathrm{~s}$ when the concentration of quercetin was five times higher than that of ketoprofen. This umbrella effect was enhanced 
(1)

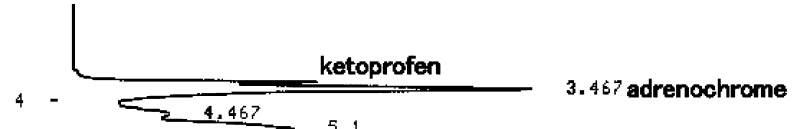

(2)

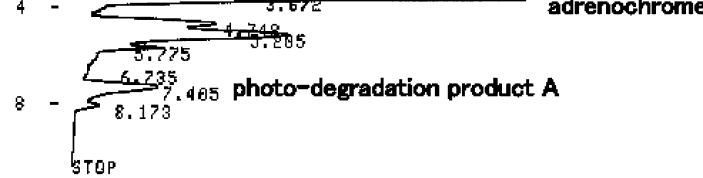

$8-\frac{6.9}{8.033} 7.5$ photo-degradation product $A$

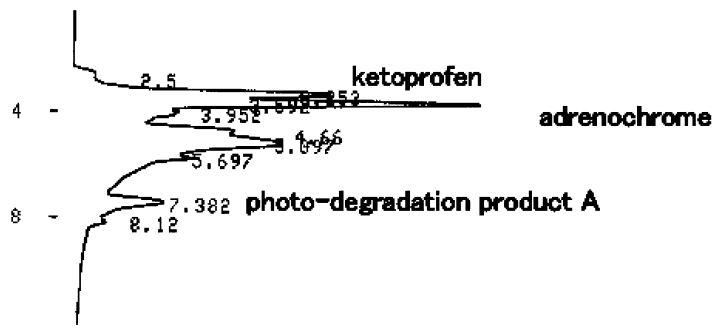

Fig. 10. HPLC Chromatograms of OPSD Experiments

(1) Ketoprofen and epinephrine solution, (2) $\alpha$-tocophenol, ketoprofen and epinephrine solution, (3) quercetine, ketoprofen and epinephrine solution. HPLC conditions: mobile phase: acetonitrile/methanol $/ 20 \mathrm{~mm}$ ammonium acetate $(30: 50: 20)$; flow rate $1.0 \mathrm{ml} / \mathrm{min}$; column: GL-PACK Nucleosil $250 \mathrm{~mm} \times 4.6 \mathrm{~mm}$ I.D.; temperature: $40{ }^{\circ} \mathrm{C}$ and detection wave length: $480 \mathrm{~nm}$.

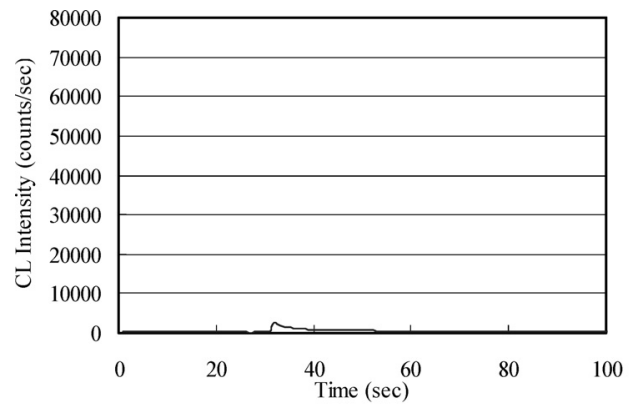

(1)

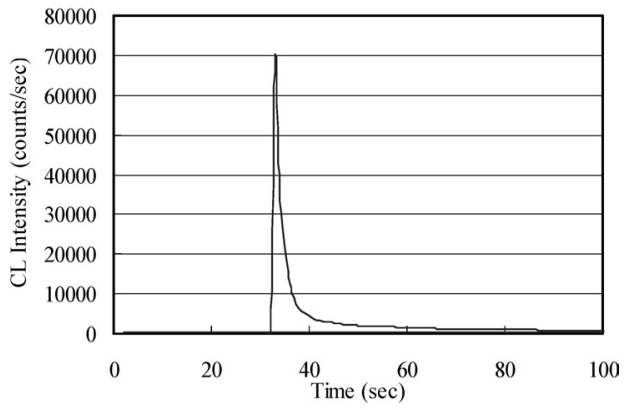

(2)

Fig. 11. The Spectra of CLA Experiments

(1) The quercetin formulation (ketoprofen + quercetin + the other excipients), (2) the control formulation (ketoprofen + the other excipients).

with increasing concentration of quercetin. Quercetin remained stable on exposure to UV. ESR experiments indicated that quercetin quenches free radicals generated from UV-ex- posed ketoprofen and that quercetin itself does not generate free radicals when exposed to UV (Fig. 7). Flavone is extremely stable to UV exposure, preventing the photo-degradation of ketoprofen; however, it does not have any quenching effect on free radicals generated from ketoprofen (Fig. 9). The ability of quercetin to quench free radicals could be attributed not to the flavone structure, but to the hydroxyl groups. The results of superoxide anion determination by the OPSD system indicated that quercetin clearly suppresses the generation of superoxide anions and photo-degradation product A, as well as the decomposition of ketoprofen. The peak height decrease of approximately $37 \%$ for adrenochrome and $31 \%$ for photo-degradation product $\mathrm{A}$ and the peak height increase of approximately $42 \%$ for ketoprofen were observed (Fig. 10, Table 3). In the CLA, the chemiluminescence was detected when the excited singlet oxygen returns to the stable triplet oxygen and excited carbonyl groups return to the stable level. ${ }^{16)}$ The results of CLA indicated that the equal mol concentration of quercetin to ketoprofen is effective to diminish $96 \%$ of generation of the singlet oxygen and excited carbonyl groups by the photoreaction of ketoprofen in the formulation (Fig. 11). As regards the photoreaction pathway of ketoprofen, we suggest that an excited carboxyl anion is primarily generated from ketoprofen by UV light energy, which could be converted into a carboxylic anion radical, generating superoxide anion and hydroxyl radical. The carboxylic acid radical is converted into the 3-(benzophenyl) ethane radical through the de-carboxylic acid pathway, and this, in turn, dimerizes to form product A. ${ }^{16)}$ Quercetin could disrupt the excitation of the carboxyl acid group, and could scavenge free radicals and active oxygen species generated from the excited carboxyl anion even if the disruption was imperfect. This means that quercetin works to suppress free radical and active oxygen species generation from the UVexposed ketoprofen, as well as to quench the free radicals. These suppressing effects of quercetin were not intercepted by the excipients of ointment's components in this study. Consequently, quercetin has high potential for use as an excipient in ketoprofen ointments to suppress phototoxicity and photosensitization by ketoprofen.

Acknowledgment We thank Mr. Hitoshi Yamauchi, Mr. Toshiyuki Takayasu, Mrs. Hiroko Matsumura and Mrs. Manami Kamijo, in Saitama Daiichi Pharmaceutical Co., Ltd., for their support with the formulations preparing.

\section{References}

1) Bagheri H., Lhiaubet V., Montasruc J. L., Chouini-Lalanne N., Drug Saf., 22, 339-349 (2000).

2) Lhiaubet V., Paillous N., Chouini-Lalanne N., Photochem. Photobiol., 74, 670-678 (2001).

3) Castelli F., De Guidi G., Giuffrida S., Miano P., Sortino S., Int. J. Pharm., 184, 21-33 (1999).

4) Radschuweit A., Ruttinger H. H., Nuhn P., Wohlrab W., Huschka C., Photochem. Photobiol., 73, 119-127 (2001).

5) Becker L., Eberlein-König B., Przybilla B., Acta Derm. Venereol., 76, 337-340 (1996)

6) Eberlein-König B., Rueff F., Przybilla B., Photodermatol. Photoimmunol. Photomed., 12, 109-113 (1996).

7) Nieto A. I., Cabre F., Moreno F. J., De Lalastra C. A., Digest. Dis. Sci., 47, 905-913 (2002).

8) Hanson K. M., Clegg R. M., Photochem. Photobiol., 76, 57-63 (2002).

9) Matsushita T., Kameda R., Photodermatol. Photoimmunol. Photomed., 17, 26-31 (2001) 
10) Milpied-Homsi B., Presse Med., 30, 605-609 (2001).

11) Albes B., Marguery M. C., Schwarze H. P., Journe F., Loche F., Bazex J., Dermatology, 201, 171-174 (2000).

12) Le Coz C. J., Bottlaender A., Scrivener J. N., Santinelli F., Cribier B. J., Heid E., Grosshans E. M., Contact Dermatitis, 38, 245-252 (1998).

13) Sugiura M., Hayakawa R., Xie Z., Sugiura K., Hiramoto K., Shamoto M., Photodermatol. Photoimmunol. Photomed., 18, 82-89 (2002).

14) Kuno Y., Numata T., J. Dermatol., 21, 352-357 (1994).

15) Boscá F., Miranda M. A., Carganico G., Mauleón D., J. Photochem. Photobiol., 60, 96-101 (1994).

16) Nakajima A., Tahara M., Yoshimura Y., Nakazawa H., J. Photochem. Photobiol. A, 174, 89—97 (2005).
17) Kawada K., Hanawa T., Nakajima S., Medical J., 37, 1610-1618 (2001).

18) Yagi K., Nakano M., "Active Oxygen-Chemistry, Biology, Medicine," ed. by Niki E., Shimazaki H., Ishiyaku Publishers Inc., Tokyo, 1987, pp. $64-85$.

19) Naru E., Moriyama M., Inomata K., Hayashi A., Arakane K., Kaji K., J. Jpn. Cosm. Sci. Soc., 26, 79-85 (2002).

20) La Farré M., Ferrer I., Ginebreda A., Figuaras M., Olivella L., Tirapu L., Vilanova M., Barceló D., J. Chromatogr. A, 938, 187-197 (2001).

21) Miao X. S., Koenig B. G., Metcalfe C. D., J. Chromatogr. A, 952, 139-147 (2002).

22) Kataoka M., Tanooka K., Ando T., Imai K., Aimoto T., Free Radical Res., 27, 419-427 (1997). 